\title{
Chronic intestinal pseudoobstruction
}

INSERM

\section{Source}

INSERM. (1999). Orphanet: an online rare disease and orphan drug data base. Chronic intestinal pseudoobstruction. ORPHA:2978

Chronic intestinal pseudo-obstruction (CIPO) is a rare gastrointestinal motility disorder characterized by recurring episodes resembling mechanical obstruction in the absence of organic, systemic, or metabolic disorders, and without any physical obstruction being detected by X-ray or during surgery. CIPO develops predominantly in children and may be present at birth. 